\author{
G. Vatcher, D. Smailus, M. \\ Krzywinski, R. Guin, J. Stott, \\ M. Tsai, S. Chan, P. Pandoh, \\ G. Yang, J. Asano, T. Olson, \\ A.-L. Prabhu, R. Coope ${ }^{1}$, A. \\ Marziali ${ }^{1}$, J. Schein, S. Jones, \\ and M. Marra \\ Genome Sciences Centre \\ BC Cancer Agency \\ ${ }^{1}$ University of British Columbia \\ Vancouver, BC, Canada
}

\section{fRFLP and fAFLP: Medium-Throughput Genotyping by Fluores- cently Post-Labeling Restriction Digestion}

BioTechniques 33:539-546 (September 2002)

\begin{abstract}
Genome-scale studies of population structure and high-resolution mapping of genetically complex traits both require techniques for accurately and efficiently genotyping large numbers of polymorphic sites in multiple individuals. Many high-throughput genotyping technologies require the purchase of expensive equipment or consumables and are therefore out of reach of some individual research laboratories. Conversely, less expensive technologies are often labor intensive so that the effort involved in typing large numbers of samples or polymorphic sites is prohibitive. Here we present a method of fluorescently post-labeling restriction digestion using standard dye-terminator sequencing chemistry so that RFLP and AFLP products can be visualized on an automated sequencer. This labeling method is efficient, inexpensive, easily multiplexed, and requires no unusual equipment or reagents, thus striking a balance between cost and throughput that should be appropriate for many research groups and core facilities.
\end{abstract}

\section{INTRODUCTION}

Restriction endonucleases have long been used in population studies of allelic variation, where the presence or absence of a cut site is indicative of a mutation in the enzyme target sequence. In genetically well-characterized organisms, diagnostic RFLPs are often used as markers for previously characterized alleles. In organisms with less well-studied genetics, the selective amplification of anonymous restriction digestion products (e.g., AFLPs) (8) has also been used with considerable success $(5,9)$. Although they are extremely powerful in the appropriate contexts, many existing RFLP and AFLP methods have limitations in throughput, sensitivity, or consumable cost that lessen their utility in many individual research laboratories. Traditionally, RFLP and AFLP products have been electrophoretically resolved on either agarose or acrylamide slab gels, with the digested fragments visualized by ethidium bromide staining or radiolabeling. Existing fluorescence-based RFLP and AFLP methods use labeled amplification primers $(1,3,4,6)$ that could cost over $\$ 100$ apiece (Research Genetics, Huntsville, AL, USA). This quickly increases the cost of an experiment using multiple primers and, in the case of RFLP, only allows the visualization of a single terminal fragment, thus losing all sequence information $3^{\prime}$ of the first restriction cut site. To increase RFLP information content and allow higher RFLP and AFLP throughput at lower costs, we have developed a simple and inexpensive method for post-labeling restriction digestion fragments using "off-the-shelf" sequencing chemistry before separation on an automated sequencer. We call this labeling method fluorescent RFLP (fRFLP).

Briefly, we amplify the target locus in a standard PCR and then digest the product with a restriction enzyme that leaves a $5^{\prime}$ overhang. This overhang acts as a template for the single base incorporation of a fluorescent dye-terminator nucleotide from a standard cycle sequencing kit. Thus labeled, the fragments are resolved on a capillary-based sequencer. Multiplexing is facilitated by the high resolution of the sequencer and its ability to read simultaneously multiple fluor wavelengths. This label- 
ing method can be applied in an AFLP context by using adapters and unlabeled primers containing a restriction enzyme recognition sequence that can be cut and fluorescently labeled after the final, selective, AFLP amplification. Fluorescent AFLP (fAFLP) products can be multiplexed by simultaneously using all of the fluor channels detected by the sequencer.

We have been working with the CEQ $^{\mathrm{TM}}$ 2000-XL Capillary-Based Sequencer and a CEQ Dye-Terminator Cycle Sequencing (DTCS) Kit (both from Beckman Coulter, Fullerton, CA, USA), but our fRFLP and fAFLP labeling methods should be feasible on any sequencing platform in which the reagents are packaged individually (i.e., not in a terminator dNTP-bufferenzyme premixture). Table 1 illustrates that the post-digestion labeling chemistry costs approximately $\$ 0.45 / \mathrm{reac}$ tion, and the electrophoretic separation costs approximately $\$ 1.35$. These costs, per site, decrease with multiplexing.

\section{MATERIALS AND METHODS}

\section{fRFLP}

Here we illustrate fRFLP by using the method to distinguish Attacin A alleles generated by gene conversion in the Attacin AB cluster of Drosophila melanogaster (7). DNA from D. melanogaster lines 2CPA 1, 2CPA 14, 2CPA 105, and 2CPA 129 was amplified using primers Att98-1 and Att98-2 (7) under standard PCR conditions. PCR was carried out for 35 cycles with 45-s annealing and extension steps at $58^{\circ} \mathrm{C}$ and $72^{\circ} \mathrm{C}$, respectively. Following amplification, $15 \mu \mathrm{L}$ each PCR product were incubated with $7.5 \mathrm{U}$ exonuclease I and 3 U shrimp alkaline phosphatase (USB, Cleveland, $\mathrm{OH}, \mathrm{USA}$ ) for $1 \mathrm{~h}$ at $37^{\circ} \mathrm{C}$ to inactivate residual primer and unincorporated nucleotides. The enzymes were then inactivated for $15 \mathrm{~min}$ at $80^{\circ} \mathrm{C}$.

We digested the PCR products with the restriction enzymes $M s p I, M s e I$, and DpnII (New England Biolabs, Beverly, MA, USA) because diagnostic polymorphisms for the Attacin $A B$ gene conversion events disrupt cut sites recognized by these enzymes and because all three enzymes cut efficiently in PCR

Table 1. Post-Digestion Cost of fRFLP and fAFLP

\begin{tabular}{|c|c|c|c|c|c|}
\hline \multirow[b]{2}{*}{ Reagent } & \multirow[b]{2}{*}{ Stage } & \multicolumn{4}{|c|}{ Per-Site Cost for Multiplexed Samples } \\
\hline & & Simplex & 3-folda & 6-folda & 9-folda \\
\hline Exonuclease I & $\begin{array}{c}\text { sample } \\
\text { preparation }\end{array}$ & 0.060 & 0.060 & 0.030 & 0.020 \\
\hline $\begin{array}{c}\text { Shrimp alkaline } \\
\text { phosphatase }\end{array}$ & $\begin{array}{c}\text { sample } \\
\text { preparation }\end{array}$ & 0.111 & 0.111 & 0.056 & 0.037 \\
\hline DTCS label & labeling & 0.163 & 0.163 & 0.082 & 0.054 \\
\hline $10 \times$ Seq. Buffer & labeling & 0.116 & 0.116 & 0.058 & 0.039 \\
\hline Separation Buffer & electrophoresis & 0.490 & 0.163 & 0.082 & 0.054 \\
\hline Separation Gel & electrophoresis & 0.550 & 0.183 & 0.092 & 0.061 \\
\hline Size Standard-600 & electrophoresis & 0.309 & 0.103 & 0.052 & 0.034 \\
\hline TOTAL (US dollars & & 1.799 & 0.900 & 0.450 & 0.300 \\
\hline \multicolumn{6}{|c|}{$\begin{array}{l}\text { aMultiplex assumes all three fluor channels are used with either one, two, or three } \\
\text { amplification products in each channel. It is assumed that digested reactions are } \\
\text { pooled for a single labeling reaction when more than one product is represented } \\
\text { in each channel and that each product queries only a single polymorphic site. } \\
\text { Estimated per-site costs vary slightly with other multiplex structures. }\end{array}$} \\
\hline
\end{tabular}

buffer, which obviates the need for a buffer change at this step. Five microliters of each PCR product were incubated overnight at $37^{\circ} \mathrm{C}$ with $5 \mathrm{U}$ each enzyme in single digestions. For simplicity, only one amplification product was digested in each of these reactions, but we have had excellent success multiplexing by pooling distinct PCRs at the digestion stage (unpublished results).

The restriction digestion reactions were labeled using components of the DCTS kit, with extra $10 \times$ sequencing buffer ordered directly from the manufacturer. The molar concentrations of the materials in this kit are proprietary, so we can only provide volume measures of reagents, but we have found this labeling reaction to be robust over a wide range of template, terminator, and DNA polymerase concentrations. For the present demonstration, each labeling reaction included $1 \mu \mathrm{L}$ digested DNA, $1.9 \mu \mathrm{L} 10 \times$ Sequencing Buffer, and $33 \mathrm{~nL}$ sequencing DNA polymerase. Only the fluorescent terminator nucleotide specific to each digestion was included in the labeling reactions (ddGTP for DpnII, ddCTP for MspI, and ddUTP for MseI). Thirty-three nanoliters of fluorescent terminator nucleotide were used in the ddCTP and ddUTP labeling reactions, but $100 \mathrm{~nL}$ were required for adequate signal with ddGTP. We added $17 \mu \mathrm{L}$ distilled water to make $20 \mu \mathrm{L}$ total volume, and the reactions were incubated for $1 \mathrm{~h}$ at $60^{\circ} \mathrm{C}$.

After the incubation, we pooled the three labeled digestions from each PCR product and added $15 \mu \mathrm{L}$ stop solution (1.2 $\mathrm{M}$ sodium acetate, $40 \mathrm{mM}$ EDTA, 4 $\mu \mathrm{g} / \mu \mathrm{L}$ glycogen). The fRFLP reactions were precipitated by the addition of 200 $\mu \mathrm{L}$ ice-cold $95 \%$ ethanol to each reaction and were then centrifuged for 20 $\min$ at $1800 \times g$. The supernatant was aspirated off, and the pellets were washed three times with $200 \mu \mathrm{L}$ ice-cold $70 \%$ ethanol and then allowed to air-dry. The dry pellets were resuspended in $40 \mu \mathrm{L}$ deionized formamide with $0.25 \mu \mathrm{L}$ CEQ DNA Size Standard-600 (Beckman Coulter) and overlayed with one drop of mineral oil. The fragments were then separated on the CEQ 2000-XL capillary-based sequencer using the default "Frag-4" method and analyzed with the fragment analysis component of the CEQ 2000-XL software, version 4.3.9 (Beckman Coulter).

\section{fAFLP}

We performed AFLP on DNA extracted from hydrothermal vent tubeworms, Ridgeia piscesae (Polychaeta: 


\section{Short Technical Reports}

Siboglinidae), collected at the Endeavor Segment of the Juan de Fuca Ridge in the northeast Pacific Ocean. Genomic DNA was extracted using a standard phenol:chloroform technique (2), and AFLP was carried out using AFLP ${ }^{\circledR}$ Analysis System I and AFLP Starter Primer kits (Invitrogen, Carlsbad, CA, USA), which follow the protocol of Vos et al. (8). DNA (250 ng) from each individual served as the starting template. For fAFLP, the final amplification was performed with a selective extension of -AGG on the EcoRI adapter primer and -CTC on the MseI adapter primer. In this example, we make use of the fact that the MseI adapter and primer contain a recognition site for the four-cutting restriction enzyme $D d e$ I.

After the selective amplification, 5 $\mu \mathrm{L}$ each AFLP amplification were incubated with $2.5 \mathrm{U}$ exonuclease $\mathrm{I}$ and $1 \mathrm{U}$ shrimp alkaline phosphatase for $1 \mathrm{~h}$ at $37^{\circ} \mathrm{C}$, followed by $15 \mathrm{~min}$ at $80^{\circ} \mathrm{C}$. The reactions were digested overnight at $37^{\circ} \mathrm{C}$ with $2.5 \mathrm{U}$ DdeI (New England Biolabs), and $1 \mu \mathrm{L}$ of the digestion was labeled with ddUTP as described earlier. Following labeling, $5 \mu \mathrm{L}$ stop solution and $60 \mu \mathrm{L}$ ice-cold $95 \%$ ethanol were added to each of the fAFLP reactions, which were then centrifuged for $20 \mathrm{~min}$ at $1800 \times \mathrm{g}$. The pellets were washed twice with $200 \mu \mathrm{L}$ ice-cold $70 \%$ ethanol and air-dried. As in fRFLP, the dry pellets were resuspended in 40 $\mu \mathrm{L}$ deionized formamide containing $0.25 \mu \mathrm{L}$ Size Standard-600 and overlayed with a drop of mineral oil. The fAFLP fragments were then resolved on the CEQ 2000-XL automated sequencer using the Frag-4 separation method.

Post-AFLP digestion with DdeI cut both in the adapter/primer sequences and at internal sites in the amplified products. All of these cut sites were subsequently labeled. In addition to supplementing genetic information about the samples, the presence of internal cut sites in larger products can bring the peaks down into the effective resolution range of the capillary sequencer (60-600 bp on default settings). However, in some cases, it may be desirable to engineer adapters and primers carrying recognition sites for rare-cutting enzymes to minimize the digestion of AFLP amplification products.

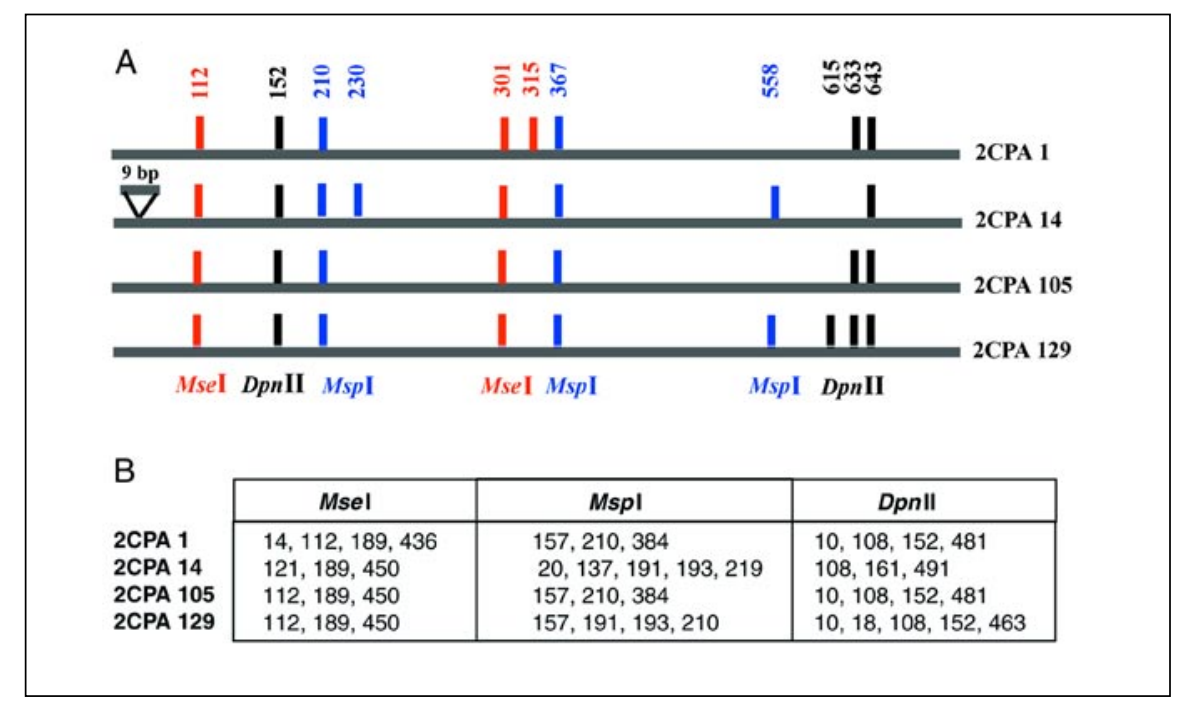

Figure 1. Digestion of the Attacin A gene of D. melanogaster with only three restriction enzymes distinguishes six polymorphisms among four alleles. (A) Schematic representation of the pattern of restriction sites in the four alleles. The numbers along the top row represent the positions of the cut sites along the 751-bp product. (B) Expected fragment sizes in the four lines, following digestion with each of the three enzymes. Fragments smaller than $60 \mathrm{bp}$ are not detected, but all of the other peaks were accurately detected and sized within $2 \mathrm{bp}$.

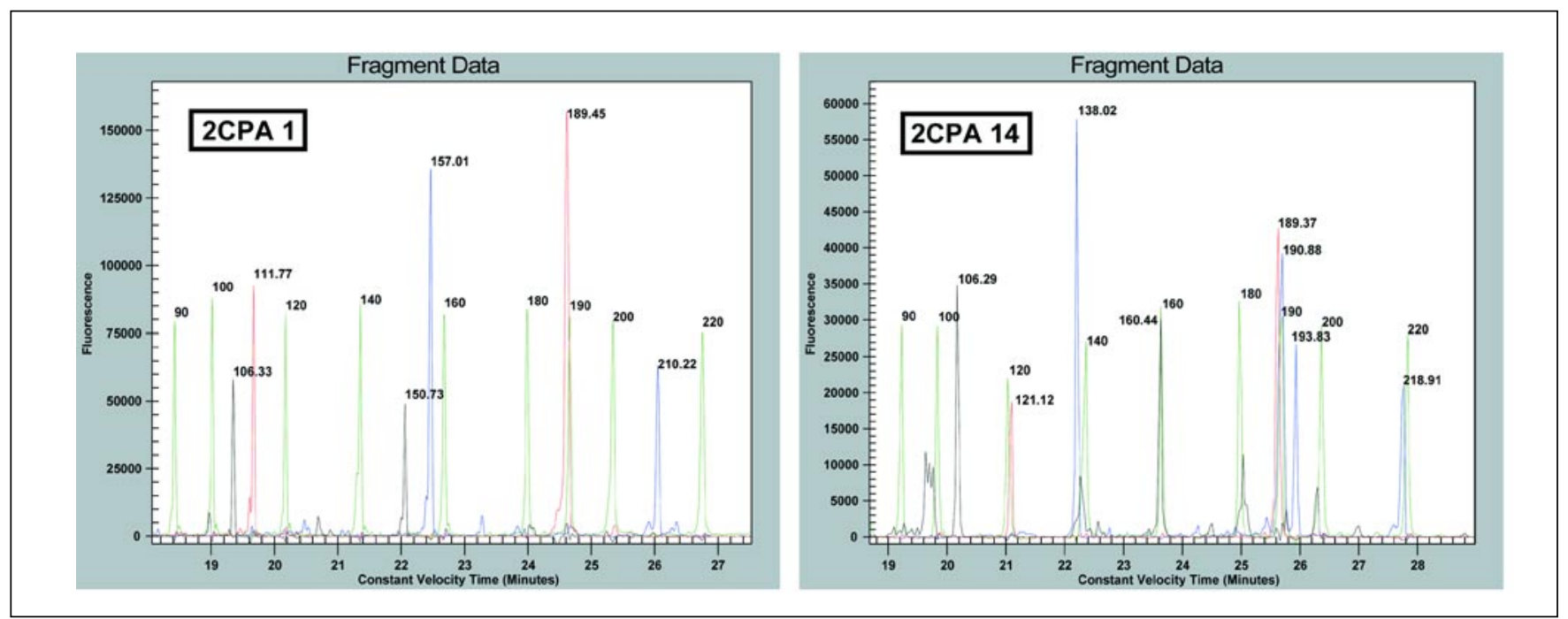

Figure 2. A zoomed-in view of chromatograms showing fRFLP peaks between 90 and 220 bp in lines 2 CPA 1 and 2CPA 14. The high resolution of the automated sequencer allows easy identification of a 9-bp insertion/deletion polymorphism [112 vs. $121 \mathrm{bp}$ in the red (T-labeled) channel; $210 \mathrm{vs.} 219$ in the blue (C-labeled) channel, and 152 vs. 161 in the black (G-labeled) channel]. Note the clear distinction between 191- and 193-base fragments in the blue channel of 2CPA 14. Green peaks are the CEQ DNA Size Standard-600 internal reference size standard. 


\section{RESULTS AND DISCUSSION}

Using only three restriction enzymes and a single electrophoretic run of fRFLP, we typed six polymorphisms, including a 9-bp insertion/deletion polymorphism, in the Attacin A gene from four lines of $D$. melanogaster (Figures 1 and 2) at a post-digestion cost of about $\$ 0.45 /$ site (Table 1 ). We also used fAFLP to generate a genomic fingerprint for the hydrothermal vent tubeworm R. piscesae (Figure 3) at a labeling cost of $\$ 0.45$, without needing to buy a fluorescently labeled primer. The total per-site cost of fRFLP varies depending on the price of the diagnostic restriction enzymes and can be reduced by higher degrees of multiplexing. We have had success in multiplexing by pooling independent reactions before restriction digestion, pooling multiple digests before fluorescent labeling (data not shown), and simultaneously

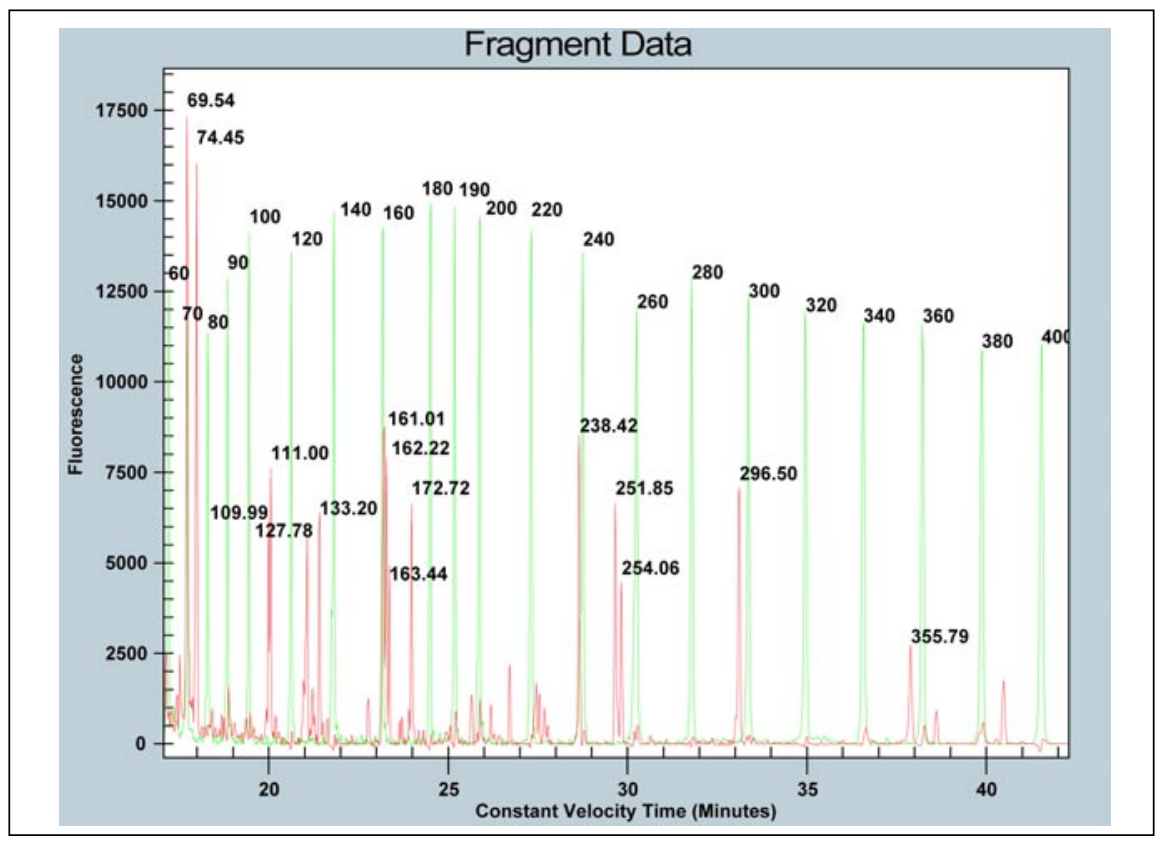

Figure 3. Chromatogram showing fAFLP peaks obtained by using DdeI to digest an unlabeled $R$. piscesae AFLP reaction and then incorporating a fluorescent ddUTP terminator nucleotide into the cut site (red peaks). Green peaks are the CEQ DNA Size Standard-600 internal reference size standard. 
electrophoresing multiple independently labeled products.

Because automated sequencers can distinguish fragments that differ in size by a single nucleotide (Figure 2 ) and can detect hundreds of fragments in a single electrophoretic run, the only practical limit to the degree of fRFLP multiplexing that can be employed seems to be in the informatics of analyzing the output. The CEQ Fragment Analysis software does not have a pattern-recognition component that would allow for the translation of highly multiplexed fRFLP peak patterns into component multi-locus genotypes, but the software does export a tab-delimited text file that lists, among other attributes, the estimated size in base pairs, and under-peak area for every peak in each fluor channel. It should be straightforward to program an independent script that analyzes the CEQ text output for expected fRFLP genotype patterns. Without the use of automated genotype calling, we have found 6-fold multiplexing, with two independent PCR products digested and labeled in each the $\mathrm{C}, \mathrm{G}$, and T channels (3-10 peaks in each channel), to provide an adequate compromise among cost per assay, throughput, and simplicity of analysis. For fAFLP analysis, a macro that generates a presence/absence table for all peaks in each fluor channel in a given sample set is available from Beckman Coulter technical support. This macro, along with other improvements that ease data analysis, is incorporated into the next generation CEQ 8000 software for more seamless fAFLP analysis.

The ability of automated sequencers to resolve extremely small size differences accurately can also be exploited in the examination of microsatellite repeats. Although we have not tested the possibility, we believe that the post-labeling procedure used in fRFLP and fAFLP could also be used in microsatellite analysis if rare-cutting restriction enzyme recognition sites are incorporated into the amplification primers. Nonoverlapping repeat size ranges and the use of multiple fluors could allow a high degree of multiplexing in microsatellite analysis. Our labeling method could conceivably even be applied to the inexpensive generation of the internal size standard required in each well for accurate sample peak sizing.

Overall, both fRFLP and fAFLP provide a simple, rapid, and inexpensive means of genotyping moderate numbers of individuals at a large number of loci with no need to order allelespecific or fluorescently labeled oligonucleotides. It is not even necessary to have prior knowledge of the polymorphisms that will be typed because both methods can be used to query anonymous or uncharacterized sequences. The cost, ease, and flexibility of these methods make them appropriate for many medium-throughput applications on a scale likely to be employed by individual research laboratories.

\section{REFERENCES}

1.Arnold, C., L. Metherell, J.P. Clewley, and J. Stanley. 1999. Predictive modeling of fluorescent AFLP: a new approach to the molecular epidemiology of $E$. coli. Res. Microbiol. 150:33-44.

2.Ausubel, F.M., R. Brent, R.E. Kingston, D.D. Moore, J.G. Seidman, J.A. Smith, and K. Struhl. 1989. Current Protocols in Molecular Biology, Vol. 1. Greene Publishing Associates and Wiley-Interscience, New York.

3.Bruce, K.D. and M.R. Hughes. 2000. Termi- nal restriction fragment length polymorphism monitoring of genes amplified directly from bacterial communities in soils and sediments. Mol. Biotechnol. 16:261-269.

4.Desai, M., A. Tanna, R. Wall, A. Efstratiou, R. George, and J. Stanley. 1998. Fluorescent amplified-fragment length polymorphism analysis of an outbreak of group A streptococcal invasive disease. J. Clin. Microbiol. 36:3133-3137.

5.Fishman, L., A.J. Kelly, E. Morgan, and J.H. Willis. 2001. A genetic map in the Mimulus guttatus species complex reveals transmission ratio distortion due to heterospecific interactions. Genetics 159:1701-1716.

6.Koeleman, J.G., J. Stoof, D.J. Biesmans, P.H. Savelkoul, and C.M. VandenbrouckeGrauls. 1998. Comparison of amplified ribosomal DNA restriction analysis, random amplified polymorphic DNA analysis, and amplified fragment length polymorphism fingerprinting for identification of Acinetobacter genomic species and typing of Acinetobacter baumannii. J. Clin. Microbiol. 36:2522-2529.

7.Lazzaro, B.P. and A.G. Clark. 2001. Evidence for recurrent paralogous gene conversion and exceptional allelic divergence in the Attacin genes of Drosophila melanogaster. Genetics 159:659-671.

8.Vos, P., R. Hogers, M. Bleeker, M. Reijans, T. van de Lee, M. Hornes, A. Frijters, J. Pot, et al. 1995. AFLP: a new technique for DNA fingerprinting. Nucleic Acids Res. 23:4407-4414.

9.Voss, S.R., J.J. Smith, D.M. Gardiner, and D.M. Parichy. 2001. Conserved vertebrate chromosome segments in the large salamander genome. Genetics 158:735-746.

We thank Cindy Jarvis and John Black at Beckman Coulter for their support of this work. We also thank Kim Juniper, the chief scientist of the May 2001 cruise to the Juan de Fuca Ridge, the crews and pilots of the $C C G V$ John P. Tully, and the ROV ROPOS for allowing tubeworm collection. This work is supported by the National Institutes of Health grant no. AI46402 to A.G.C. Tubeworm collections were supported by the $\mathrm{Na}$ tional Underseas Research Program grant no. UAF01-0042 to Stephen Schaeffer, Charles Fisher, and Stéphane Hourdez. Address correspondence to . Brian P. Lazzaro, Department of Molecular Biology and Genetics, 107 Biotechnology Building, Cornell University, Ithaca, NY 14853, USA. e-mail: bplazzaro@psu.edu

Received 13 March 2002; accepted 22 May 2002.

Brian P. Lazzaro, Bonnielin K. Sceurman, Susan L. Carney, and Andrew G. Clark Pennsylvania State University University Park, PA, USA 